\title{
Lumen
}

Selected Proceedings from the Canadian Society for Eighteenth-Century Studies

\section{Le secret dans les romans-mémoires de Prévost}

\section{Michèle Bokobza Kahan}

Volume 37, 2018

URI : https://id.erudit.org/iderudit/1042222ar

DOI : https://doi.org/10.7202/1042222ar

Aller au sommaire du numéro

Éditeur(s)

Canadian Society for Eighteenth-Century Studies / Société canadienne d'étude du dix-huitième siècle

ISSN

1209-3696 (imprimé)

1927-8284 (numérique)

Découvrir la revue

Citer cet article

Bokobza Kahan, M. (2018). Le secret dans les romans-mémoires de Prévost.

Lumen, 37, 35-51. https://doi.org/10.7202/1042222ar d'utilisation que vous pouvez consulter en ligne.

https://apropos.erudit.org/fr/usagers/politique-dutilisation/ 


\title{
Le secret dans les romans-mémoires de Prévost
}

\author{
MichÈLE BoKobZa KaHAN \\ Université de Tel-Aviv
}

Le secret occupe une place centrale dans les romans-mémoires de Prévost, quel que soit l'angle d'analyse abordé. Les schémas narratifs, énonciatifs et diégétiques s'entremêlent et se nourrissent mutuellement à des degrés divers autour d'une notion qui est appréhendée ici dans son sens largel.

Dans Le doyen de Killerine comme dans Histoire d'une Grecque moderne, pour ne citer que les exemples les plus probants, la perspective narratologique montre comment les processus de communication qui se construisent entre le je narrant et le je narré soulèvent des questions reliées aux conflits psychiques d'un moi instable souvent contradictoire. La subtilité avec laquelle Prévost utilise des transitions entre registre narrant et registre narré, apporte à la littérature de son temps un élément de subjectivité que je relie à la question du secret dans sa dimension affective et morale. Secrets de l'inconscient que l'on ne maîtrise pas mais qui se dégagent des interstices du texte, secrets intentionnés, voulus, programmés, rationalisés, reliés eux aussi à une psyché mais plus concrètement présents dans la diégèse, secrets reliés aux relations qui se tissent entre les personnages, relations définies et appréhendées à travers ces secrets, qui déterminent une progression ou une régression communicationnelle. L'intrigue se construit autour de malentendus et de quiproquos dus aux mensonges, aux dissimulations,

1. L'étymologie du mot (à sa source le verbe «cerno» qui signifie «cribler, passer au crible») révèle la dimension de sélection, de séparation ou de mise de côté d’une chose que l'on souhaite détenir. 
aux déguisements, aux silences des personnages, autant d'éléments qui remplissent un rôle déterminant dans leur destin. Cleveland (Le philosophe anglais ou Histoire de Monsieur Cleveland), les proches du doyen de Killerine, des Grieux et Manon, ou Perés, le compagnon fidèle du commandeur (La jeunesse du commandeur ou Mémoires pour servir à l'Histoire de Malte), sont les victimes directes ou indirectes de secrets qu'ils cachent ou qui leur sont cachés. Enfin, Prévost a su pratiquer une écriture suggestive qui entretient une ambiance d'équivoque féconde entre l'instance auctoriale, le narrateur autodiégétique et le lecteur, comme en témoignent les romans déjà cités, l'Histoire du chevalier des Grieux et de Manon Lescaut ou La jeunesse du commandeur.

Les déclinaisons diverses du secret, tant au plan thématique (comportements et objets reliés au secret), qu'au plan narratif (ellipses, non-dits, court-circuitages communicationnels), témoignent d'un art du récit; elles participent également d'une réflexion complexe sur les chemins du savoir et de la connaissance de soi que l'on ne peut envisager uniquement sous un angle moraliste imprégné d'une vision religieuse chrétienne de la condition humaine ${ }^{2}$. Perçu au XVII ${ }^{\mathrm{e}}$ siècle comme une stratégie politique ${ }^{3}$, le secret se déploie avec Prévost bien au-delà de la sphère des affaires publiques vers celle intime des passions. Ces aspects du secret soulèvent dans leur dynamique permanente des questions d'ordre moral et philosophique parce que le mouvement vers la vérité est indissociable d'un geste d'évitement de cette même vérité vers laquelle l'on aspire, et cette dynamique du secret préserve une part de mystère et de doute dans le récit, qui place l'œuvre romanesque de Prévost de plein pied dans la modernité des Lumières ${ }^{4}$.

2. Alan J. Singerman consacre un ouvrage à l'impact de la vision chrétienne de la conduite humaine dans l'étude des passions chez Prévost, Labbé Prévost. Lamour et la morale, Genève, Droz, 1987.

3. Voir Ronald W. Tobin, «Le secret dans les tragédies de Racine», Revue d'histoire littéraire de la France, 107, 4, 2007, p. 887: «Savoir est pouvoir, et le secret est toujours un savoir.»

4. Sur les ambiguïtés de la pensée des Lumières, voir l'incontournable ouvrage de Robert Mauzi, Lidée du bonheur dans la littérature et la pensée françaises au dixhuitième siècle, Paris, Albin Michel, 1979. 


\section{Le secret dans la diégèse}

Lunivers romanesque prévostien regorge de secrets qui bouleversent le cours des événements, construisent et détruisent des alliances, des amours, des amitiés, provoquent et entretiennent le suspens. Prévost met en scène le secret dans un geste spectaculaire qui est proche de celui analysé par Arielle Meyer5. Les audiences secrètes (Histoire d'une Grecque moderne, Campagnes philosophiques), les rendez-vous amoureux clandestins (Mémoires d'un honnête homme), les identités dissimulées ou échangées (plusieurs épisodes dans Histoire du chevalier des Grieux et de Manon Lescaut), les déguisements, les complots, et autres formes de secrets, entraînent le lecteur dans les rets d'histoires souvent sombres, riches en rebondissements, et le rapprochent de personnages malheureux, trahis et trompés. Enfin, s'il est conseillé de ne pas garder de secrets, mieux vaut parfois se taire. Ainsi, le secret du faux statut matrimonial de des Grieux et de Manon Lescaut entraîne, une fois dévoilé, la fin tragique que l'on connaît.

Ressort romanesque efficace, le secret participe également de la construction psychologique des personnages et de leurs rapports avec autrui. Dans Le doyen de Killerine, secrets et conspirations nourrissent l'essence de l'action dramatique et travaillent la dynamique relationnelle des personnages. Les frères et leur sœur fomentent des complots, ne se font jamais entièrement confiance, mentent et sèment le soupçon parmi leurs proches. Il suffit du retour imprévu du Doyen, après une visite écourtée à Saint-Germain, pour constater l'ampleur des secrets familiaux:

En entrant dans la cour j’aperçus quelques laquais d'une livrée inconnue, deux carrosses et des chevaux qu'on achevait de dételer. J'avance vers la maison. On me reconnaît, et j'entends aussitôt le bruit des fenêtres et de la porte des salles qu'on fermait avec la dernière précipitation. J'en croyais à peine mes oreilles et mes yeux. Que prétendent-ils? disais-je; voudraient-ils m'exclure tout à fait du logis? J'entre .

Cet exemple illustre la manière dont le secret revêt diverses formes dans le roman. Présent en toute chose, en tout lieu, dans le suivi des événements comme dans les replis du cœur et de la conscience, le

5. Arielle Meyer, Le spectacle du secret, Genève, Droz, 2003.

6. Le doyen de Killerine, histoire morale, dans (Euvres de Prévost, Grenoble, Presses universitaires de Grenoble, 1977, t. 3, p. 43. 
secret nourrit les amours des jeunes gens et sert leurs desseins. Mais victimes de leurs manigances et simulacres, soumis à des circonstances extérieures imprévues, les personnages sont contraints de révéler leurs secrets. Patrice, forcé d'épouser Sara Fincer, avoue au Doyen la promesse faite à de Presses, amoureux de sa sœur Rose, ainsi que l'engagement de ce dernier à retrouver Mlle de L., dont Patrice est amoureux. Ce n'est qu'à la veille de son départ d'Irlande pour la France que Killerine reçoit les confidences de son frère malade qui, effondré, s'exclame: «Hélas! Quel détour dois-je employer pour vous découvrir un secret que les circonstances ne me permettent plus de vous cacher $^{7}$ ? Rose, quant à elle, n'aime ni de Presses ni Linch, mais un inconnu avec qui elle entretient une correspondance secrète ignorée de ses frères. Ces secrets, révélés un peu trop tôt ou un peu trop tard, provoquent les catastrophes décrites par le prélat, qui est lui-même un grand amateur de secrets dont les motivations relèvent d'un désir inavoué et sans doute inconscient d'emprise et de domination quasi pathologique ${ }^{8}$.

Ainsi, le secret brise la fluidité de la communication entre les protagonistes tel un écran qui rompt la spontanéité et la transparence des échanges. Dans Cleveland, Fanny préfère taire ses soupçons et dissimuler sa jalousie. Mais le chagrin que provoque la supposée liaison amoureuse entre son époux et Mme Lallin, la pousse à détruire sa vie. Les malheurs qui s'abattent sur sa famille auraient pu être évités si elle avait parlé en toute confiance à son mari. Toutefois, comme dans Le doyen de Killerine, le secret se niche dans les tréfonds d'une âme jalouse. Les coups de théâtre et les effets de dramatisation que provoquent les secrets non partagés de Cleveland et de Fanny participent de la construction de la diégèse. Mais le secret ne bouleverse pas seulement la vie de Fanny, malheureuse victime des «artifices et inventions de Gelin" », il agit également sur les conflits psychiques qui torturent la jeune femme désespérée, incapable de juger avec lucidité de la conduite de son entourage parce qu'elle-même ignore les secrets de son âme:

7. Ibid., p. 105 .

8. Voir Erik Leborgne, "L'humour noir chez Prévost: le cas du Doyen de Killerine », dans C. Bournoville, C. Duflo, A. Faulot et S. Pelvilain (éd.), Prévost et les débats d'idées de son temps, Louvain, Peeters, 2015, p. 171-191.

9. Le philosophe anglais, dans Euvres de Prévost, op. cit., t. 2, p. 383. 
Je ne découvrais pas clairement ce qui se passait dans mon cœur, mais j'y sentais depuis la Corogne des agitations qui ne ressemblaient point à celles que j'avais éprouvées. Je voulais les démêler sans être interrompue. Je portais dans mon propre sein un secret qui m’était inconnu à moi-même et qu'il me semblait important d'approfondir. [...] Que croyez-vous, ma sœur, que je trouvai dans ce cœur si longtemps inconsolable, à la place de la jalousie, de la fureur, et de toutes les mortelles passions qui l'avaient déchiré? J'y trouvai l'amour, avec toutes ses tendresses et ses plus ardents transports ${ }^{10}$.

Fanny réalise que l'amour est une flamme vivante qui donne sens à sa vie, et elle comprend que cet amour a été empoisonné par sa jalousie secrète. Il en va de même pour Cleveland qui de son côté favorise par sa conduite le malentendu catastrophique entre lui et son épouse. Il a longtemps gardé secret son amour pour Fanny; il a caché le voyage de Mme Lallin avec lui pour l'Amérique; il préfère se taire sur le danger de l'épidémie qui décime les Abaquis; il n'avoue pas le désespoir qui l'envahit à l'annonce du départ de Fanny; il dissimule son état d'homme marié quand il tombe amoureux de Cécile. À cette liste, il faudrait ajouter également les secrets reliés aux personnages secondaires du roman, Bridge, Gelin, Monmouth, et bien d'autres encore.

L'histoire plus intime qu'expose l'ambassadeur dans L'histoire d'une Grecque moderne, repose également sur de nombreux secrets. L'évocation d'une maison de campagne destinée à la satisfaction discrète des désirs du diplomate français contribue à la construction d'un monde feutré et mystérieux que l'on associe à l'ambiance libertine décrite par Crébillon fils, Bastide ou Laclos. De plus, l'espace géoculturel de l'Orient où se déploie le récit renvoie à un imaginaire peuplé d'enceintes closes à l'abri desquelles pullulent, en ces intrigues latérales qu'affectionne Prévost, mensonges, infidélités et tromperies. Ainsi de l'aventure de la compagne de Théophé, ainsi des entreprises amoureuse de Synèse, frère présumé de Théophé, ainsi encore des manigances de l'esclave Béma, et des amours tumultueuses de Maria Rezati et du chevalier de Malte. En bref, les topoï libertins qui contredisent les liens passionnels évoqués apparaissent jusqu’au terme des aventures de l'ambassadeur dont l'amour pour l'esclave qu'il a libérée du sérail ne ressemble en rien au coup de foudre d'autres héros prévostiens

10. Ibid., p. 426 . 
(Renoncour et Selima dans Mémoires et aventures d'un homme de qualité, Cleveland et Fanny dans Le philosophe anglais, des Grieux et Manon, déjà évoqués, etc.).

En résonance avec les secrets qui participent largement de la construction de l'intrigue et du déroulement des événements, des éléments d'ordre narratif déploient une partition riche en nuances autour du secret et complexifient de la sorte le dispositif énonciatif du récit mémoriel.

\section{Les coulisses secrètes du projet autobiographique dans les romans-mémoires de Prévost}

Prévost joue en virtuose du va-et-vient d'un je narré et d'un je narrant pour construire des labyrinthes mentaux et affectifs complexes. À l'aide de mécanismes d'amplification et de démultiplication qui épaississent les personnages, leur psyché et la nature de leurs relations interpersonnelles, l'auteur installe un dispositif énonciatif qui laisse voir comment le narrateur, conscience à la fois critique et mensongère, devient un personnage à part entière de la diégèse.

Le projet autobiographique qui gouverne les romans-mémoires du $\mathrm{XVIII}^{\mathrm{e}}$ siècle s'appuie sur le schéma narratif d'une énonciation double ${ }^{11}$. Lacteur devenu spectateur se présente au lecteur dans la simultanéité de l'action et de la réflexion. En tant que narrateur autodiégétique, il raconte les événements de sa jeunesse, et développe à travers ce travail d'écriture une forme de sagesse critique bénéfique. Les aléas de la vie, le danger des passions, les erreurs humaines souvent catastrophiques, sont relatés par le prisme distancié de celui qui a vécu et qui a compris ce qu'il ignorait au moment de l'action. Lucide, il prend la plume pour comprendre les erreurs de jadis. «Le double registre du récit et du regard sur le récit ${ }^{12}$ », pour reprendre l'expression de Jean Rousset, met en relief l'écart temporel qui sépare la période des événements diégétiques de celle de leur narration. Cet écart, loin de garantir l'authenti-

11. Jean Rousset écrit: «la première personne ne suffit pas à définir le romanmémoires, car il la partage avec le roman-journal intime, dont la formule sera tardive, et avec le roman par lettres, qui se diffuse au même moment; il y faut un second trait: la perspective temporelle : le narrateur regarde son histoire de loin, il en connaît l'issue, il ne se saisit que rétrospectivement» (Narcisse romancier. Essai sur la première personne dans le roman, Paris, José Corti, 1986 [1972], p. 127).

12. Jean Rousset, Forme et signification, Paris, Corti, 1962, p. 50. 
cité d'un témoignage tardif, se nourrit à la source d'une mémoire sélective et d'une subjectivité ouvertement assumée puisque le narrateur se raconte tel qu'il «s'est vu vivre ${ }^{13}$ », ou tel qu'il veut se montrer à son destinataire et à son lecteur. Si dans le contexte du champ littéraire du XVIII ${ }^{\mathrm{e}}$ siècle, l'expérience d'un héros assagi semble assurer la dimension morale d'un roman d'aventures libertines, et, ce faisant, obéit à l'impératif didactique qui ouvrirait l'accès aux genres nobles ${ }^{14}$, l'aspect trompeur du roman-mémoires souligné par René Démoris ${ }^{15}$ et par Jean Rousset ${ }^{16}$, pour ne citer qu'eux, a été puissamment exploité par un auteur comme Prévost qui ne se contente pas des potentialités convenues d'une énonciation simultanée.

Dans ses romans-mémoires, un je est placé au centre de l'espace romanesque, et à travers sa parole, il s'achemine, contre toute attente, vers des sentiers tortueux qui mènent à la confusion et au doute. Le travail de mémorisation et d'introspection s'ouvre sur une enquête de la psyché humaine, ses méandres et ses profondeurs. L'exercice mémoriel, loin de construire une identité stable et identifiable, favorise un aveuglement de soi qui bien souvent s'ignore, comme c'est le cas de l'ambassadeur à Constantinople. De plus, la confrontation avec l'altérité, la sienne, en premier, et celle des autres, en second, n'est jamais sereine, comme en témoignent les tergiversations de Cleveland. Enfin, remplacer le démiurge auctorial omniscient par une voix actorielle présente et active au sein de l'histoire, c'est avouer la valeur relative de la vérité et mettre en scène les limites d'une instance auctoriale privée d'un don d'ubiquité et de toute puissance divine.

L'ambiguïté des déclarations d'ouverture prononcées par le narrateur interpelle le lecteur qui remarque d'emblée les injonctions contradictoires du projet autobiographique. Le déroulement chronologique de la réalité passée qui donnerait au moment de l'écriture un sens historique à ce qui était auparavant perçu comme le fruit de prédéterminations, de forces extérieures invisibles ou du hasard, n'est

13. Jean Rousset, Narcisse romancier, op. cit., p. 23.

14. Sur le sujet, je renvoie à l'ouvrage pionnier de Georges May, Le dilemme du roman au XVIII siècle. Étude sur les rapports du roman et de la critique (1715-1761), Paris, Presses universitaires de France, 1963.

15. René Démoris, Le roman à la première personne. Du classicisme aux Lumières, Genève, Droz, 2002 [1975].

16. Jean Rousset, Narcisse romancier, op. cit. 
nullement garanti. La dimension didactique explicitement exposée fonctionne parfois en trompe-l'œil, c'est-à-dire qu'au lieu de mener à une meilleure compréhension de la psyché humaine, le processus de remémoration et d'écriture conduit le personnage narrateur à se retrouver prisonnier de ses propres contradictions. Le besoin de justification (Les campagnes philosophiques), le travail d'introspection grâce auquel le narrateur cherche à s'affranchir d'une obsession douloureuse (Histoire d'une Grecque moderne), le travail de mémorisation capable de donner un sens nouveau aux malheurs d'une vie (Le philosophe anglais ou Histoire de Monsieur de Cleveland, Mémoires d'un honnête homme), le désir d'un échange, d'une complicité amicale, la recherche d'empathie et d'écoute consolatrice qui pousse le personnage à une confidence tendancieuse (Les aventures du chevalier des Grieux et de Manon Lescaut), l'invitation à l'émotion (La jeunesse du commandeur), constituent des mobiles légitimes qui cachent toutefois d'autres pulsions, souterraines, mystérieuses, et, sans doute, moins honnêtes. Examinons quelques exemples.

Dans Le doyen de Killerine, le personnage narrateur éponyme annonce la visée morale de ses mémoires en ces termes:

Je réponds qu'en attribuant tant de vertu à l'envie de se rendre utile, je lui suppose pour fondement toutes les qualités naturelles et acquises qui sont nécessaires d'ailleurs pour former un bon écrivain [...] le motif qui me le fait entreprendre est tel du moins que je l'ai dit; et je suis si persuadé qu'il est propre à former de bons historiens lorsqu'il se trouve soutenu des qualités qui me manquent, que je le crois même capable de suppléer à la médiocrité des miennes. S'il ne me communique point la beauté de l'imagination, qui est un présent de la nature, et les grâces du style, qui sont ordinairement des effets de l'art, il me rendra sincère dans mon récit, modeste dans mes expressions, et non seulement sage et raisonnable, mais solidement chrétien dans les principes de ma morale; il m'empêchera d'approuver ou de flatter le vice, dans les personnes même qui m’ont été les plus chères; et il me fera tourner les événements les plus profanes à l'instruction de la jeunesse, à l'édification de tous les âges et de toutes les conditions, et par conséquent à l'honneur du Ciel et à l'avantage de la société humaine ${ }^{17}$.

De la posture affichée par le Doyen se dégage un projet pédagogique utilitaire qui contredit le pacte de vérité prononcé dans un même

17. Prévost, Le doyen, op. cit., p. 14. 
souffle dans le passage cité. Tout en condamnant les mémorialistes et les historiens qui falsifient la vérité empirique pour des raisons personnelles, lui-même s'engage sur une route où il faudrait transformer la réalité pour être en accord avec les principes de morale convenus. L'aspect ambivalent des propos du doyen apparaît tout au long d'un roman habité par un «humour noir ${ }^{18}$ ", pour reprendre les propos d'Erik Leborgne. Les agissements répétés de personnages extravagants soulignent les effets poétiques d'une écriture inquiète qui serait à l'écoute de mouvements de mauvaise foi, de déresponsabilisation et de déni. Aussi, la promesse d'édifier le lecteur prononcée par le doyen au nom d'une morale religieuse ne se réfère-t-elle que très partiellement à une réalité biographique, comme l'a soutenu Jean Sgard ${ }^{19}$. La portée du préambule cité plus haut est bien ironique et donc critique. Elle nous prépare à la rencontre avec un univers romanesque où les dimensions grotesque et pathétique se rapprochent jusqu'à se confondre; dans les deux strates du double registre, le doyen se présente comme un personnage dédouané de toute responsabilité alors même que cette responsabilité ne fait aucun doute. Au nom du devoir et de l'amour fraternel, le doyen assure son pouvoir sur les siens et maintient une relation d'emprise qui atteint parfois un haut degré de sadisme, comme en témoigne le passage suivant:

Pendant que je gémissais sur sa [Georges] conduite, le Ciel m’inspira l'envie de le punir en lui enlevant Rose au milieu même de ses plaisirs.

[...] Je regardai Rose comme une victime toute parée que j'avais dérobée heureusement au sacrifice de sa vertu, et que je ramenais en triomphe ${ }^{20}$.

Prévost met en scène un décalage quasi permanent entre les intentions (bonnes?) du héros narrateur et les catastrophes qu’elles déclenchent. Le manque de clairvoyance psychologique de celui-ci et les intérêts très matériels qu'il cache derrière des prérogatives d'ordre moral, interpellent le lecteur confronté à une instance énonciative autoritaire qui ne se remet jamais en cause. Erik Leborgne parle à ce sujet d'«une

18. Erik Leborgne, op. cit.

19. Jean Sgard a évoqué la conjoncture biographique qui expliquerait l'attitude conciliante de l'auteur à l'égard de destinataires jésuites, dont la protection lui était nécessaire. Concilier, comme il l'affirme dans la préface, les «maximes du monde» avec «celles du christianisme », c'est montrer patte blanche aux instances ecclésiastiques; voir Prévost romancier, Paris, José Corti, 1989 [1968], p. 319.

20. Prévost, Le doyen, op. cit., p. 37-38. 
fermeture totale sur le moi », d'une «posture narcissique inébranlable $»^{21}$, autant de caractéristiques qui invitent le lecteur à une réévaluation permanente de la bonne foi du héros et à une méditation sceptique sur ses principes de morale. Et cette invitation à développer une lecture critique que le mémorialiste lui-même ne parvient pas à engager dans son propre récit, jette une lumière sur la force avec laquelle le romancier parvient à décrire la profonde confusion psychique du doyen ${ }^{22}$.

Un doute critique similaire s'empare du lecteur au début de l'Histoire d'une Grecque moderne. Des mots comme «franchise», «aveuglement», «amertume», témoignent d'une émotivité coléreuse et rancunière incapable de juger les faits avec objectivité. Alors que l'ambassadeur / diplomate se présente dès la seconde phrase de ses mémoires comme la victime d'une "passion violente», il s'emploie à souligner à maintes reprises le caractère insidieux et graduel d'un sentiment ambigu, ce qui nous éloigne de la représentation du coup de foudre traditionnel. Sa réputation et son tact diplomatique l'incitent à surveiller son langage et à ménager sa conduite, surtout en amour. Il se plie aux mœurs orientales quand il s'abstient de toute liaison ostentatoire et préfère un libertinage discret. Si le regard rétrospectif du narrateur persiste à interpréter les événements passés par le prisme d'une subjectivité déformante qui impose une version amoureuse de la relation, le portrait inaugural du héros esquisse l'image d'un diplomate brillant, homme du monde habile à nouer des liens d'amitié avec les hautes personnalités que sa charge l'amène à côtoyer.

Le pacte autobiographique qu'instaure le narrateur éponyme avec son lecteur dans Le philosophe anglais ou Histoire de Monsieur Cleveland prend également une tournure étrange dès les premières lignes du roman. La formule désormais célèbre de «pacte autobiographique », proposée par Philippe Lejeune, se réfère, on le sait, à l'autobiographie dans le contexte du champ littéraire et au contrat établi entre un auteur et un lecteur empirique ${ }^{23}$. Elle demeure tou-

21. Erik Leborgne, op. cit., p. 188.

22. Alan J. Singerman écrit à ce sujet: «le doyen est lui-même victime d'une profonde confusion, qui relève à la fois d'un système de valeurs obsessionnel et d'une totale méconnaissance du cœur humain» (op. cit., p. 165). Il place toutefois cette constatation au sein d'un débat religieux.

23. Philippe Lejeune, Le pacte autobiographique, Paris, Seuil, 1975. 
tefois pertinente au plan de la diégèse, quand on l'introduit dans le monde fictionnel du roman-mémoires pour analyser les rapports qui se tissent entre les acteurs de papier (auteur, narrateur et personnage ont une même identité) du dispositif énonciatif. La raison d'être du projet autobiographique réside traditionnellement dans l'expérience de la remémoration, de l'inscription testimoniale, de la préservation des souvenirs et du travail introspectif. Or, ce travail psychique place un narrateur comme Cleveland dans un état nostalgique, voire dépressif. Au fil des pages, le récit trahit bien souvent l'axe chronologique des événements pour plonger dans l'univers des sentiments et des émotions qui agitent tantôt le personnage, tantôt le narrateur. Cleveland confirme son humeur sombre dans un passage comme celui-ci : "C'est le goût de la tristesse que je consulte bien plus que les règles de la narration et que les devoirs de l'historien ${ }^{24}$. À cet $^{-}$ égard, Sade fait preuve de perspicacité quand il compare Prévost à un «Richardson français ${ }^{25} »$. Deux décennies avant la traduction de Clarisse Harlove par Prévost, et quelque trente ans avant La nouvelle Héloïse, Prévost inaugure le projet esthétique d'«une écriture au présent ${ }^{26}$ », dans le sens qu'il faut donner à un récit le long duquel le narrateur demeure pleinement conscient du travail psychique qui s'accomplit à travers sa prise de parole. Un travail qui, loin de libérer le sujet de ses angoisses passées, hante durablement son imaginaire ${ }^{27}$. L'incipit de ce «trop long ${ }^{28}$ » roman mérite une attention particulière dans le cadre de notre réflexion sur le «secret» prévostien. Prévost met en scène un mémorialiste qui insiste sur la relation très particulière qui se tisse entre un «lui », auteur de papier, et les mots qui surgissent de sa mémoire :

24. Prévost, Le philosophe anglais, op. cit., p. 241.

25. Sade, Idées sur les romans, dans Euvres complètes, Paris, Cercle du livre précieux, 1975, t. 10, p. 13.

26. Samuel Richardson, cité dans l'introduction de Lettres anglaises ou Histoire de Miss Clarisse Harlove, éd. de Shelly Charles, Paris, Desjonquères, 1999, p. 12.

27. Voir l'étude de Jean Sgard, «De Prévost à Sade», dans Eighteenth Century Fiction, 1, 1, 1988, p. 25-36: "Ce que certainement Sade a admiré le plus en Prévost, c'est le génie de l'intrigue “implexe", cette sorte de développement à l'infini à partir d'un noyau productif» (p. 29).

28. Marc Escola, «Longueur de Cleveland», dans Jean-Paul Sermain (éd.), Cleveland de Prévost, l'épopée du XVIII siècle, Paris, Desjonquères, 2006, p. 181. 
J'expose l'histoire de mes malheurs au public. Ne me demandera-t-on pas quelle sorte de plaisir peut trouver un misérable à se rappeler le souvenir de ses peines par un récit qui ne saurait manquer d'en renouveler le sentiment? Ce ne peut être qu'une personne heureuse qui me fasse cette question, car tous les infortunés savent trop bien que la plus douce consolation d'une grande douleur est d'avoir la liberté de se plaindre et de paraître affligé. Le cœur d'un malheureux est idolâtre de sa tristesse autant qu'un cœur heureux et satisfait l'est de ses plaisirs. Si le silence et la solitude sont agréables dans l'affliction, c'est qu'on s'y recueille, en quelque sorte, au milieu de ses peines, et qu'on y a la douceur de gémir sans être interrompu. Mais c'est une consolation plus douce encore de pouvoir exprimer ses sentiments par écrit. Le papier n'est point un confident insensible, comme il le semble: il s'anime en recevant les expressions d'un cœur triste et passionné; il les conserve fidèlement au défaut de la mémoire; il est toujours prêt à les représenter; et non seulement cette image sert à nourrir une chère et délicieuse tristesse, elle sert encore à la justifier. Je commence donc mon récit ${ }^{29}$.

La performance narrative d'un héros, frappé par le mauvais sort, est mise en exergue ici, comme pour mieux préparer le lecteur au récit d'une voix singulière et sensible qui cherche moins à livrer un témoignage authentique qu'à mener une enquête personnelle sur le devenir de son moi. Le héros parle du réconfort qu'il trouve dans l'acte d'écrire, de l'apaisement que lui procure le travail créateur. Mais Cleveland mémorialiste demeure prisonnier des hésitations et des doutes psychologiques qui obscurcissent l'ouverture désirée vers une meilleure compréhension de soi et de l'autre. Lorsqu'à la fin du roman, son ami et confident, le comte Clarendon, s'interroge sur la nature de ce que Cleveland «a à combattre dans l'esprit ou dans le cœur », la réaction de ce dernier est violente:

Cette proposition m’effraya. À quels retours ne m’obligeait-elle pas sur moi-même, et quelle apparence de pénétrer tout d'un coup un chaos sur lequel j’avais évité de tourner les yeux depuis si longtemps? Hélas, cher comte! Lui dis-je, comment prétendez-vous que je puisse vous apprendre ce que je m'efforce continuellement d'ignorer? Songez-vous que depuis plusieurs années toute mon étude est de fuir la vue de moi-même, par la crainte d'y trouver sans cesse un ennemi $[\ldots]^{30}$ ?

29. Prévost, Le philosophe anglais, op. cit., p. 17.

3o. Ibid., p. 1056 . 
C'est bien la raison d'être du roman qui est mise en doute dans ce passage. Malgré les promesses de bonne foi formulées au début du livre, le projet autobiographique butte sur l'écueil d'une subjectivité insaisissable, incontrôlable, aveugle à elle-même, qui convoque souvent le secret pour s'ignorer.

Ces quelques exemples montrent comment le narrateur, qu'il soit homme d'église, diplomate ou philosophe, évolue dans un dire problématique: les mensonges, les secrets et les non-dits, autant d'éléments perturbateurs qui se trouvent à la croisée des registres de la narration, du récit et de l'histoire et dont l'entrelacement complique l'interprétation et problématise la question de la vérité et de la connaissance.

\section{Vérité et secret}

Pris dans les rets d'un système narratif subtil et retors, le lecteur ne peut décider si l'entreprise autobiographique relève d'une quête de la vérité ou de la réhabilitation, vaine, d'un Moi irrémédiablement déchu. L'ambassadeur de la Grecque moderne ne réussit pas, malgré ses efforts, à élucider l'énigme de son histoire d'amour: qui des deux est le coupable? Lui qui aurait abusé de ses droits, ou Théophé, l'esclave affranchie, qui lui aurait menti? Alors que son intention première était de soumettre son dossier au verdict du lecteur («j’abandonnerai le jugement à mes lecteurs »), le vieillard grabataire demeure la victime de ses propres secrets. S'il prétend présenter en toute objectivité le récit de sa relation avec Théophé, personnage mystérieux, il révèle entre les lignes le caractère paranoïaque d'un amour déçu. Frappé de cécité, il peint le tableau d'une psyché paranoïaque incontrôlable. Le ressentiment haineux qu'il éprouve envers cette femme qu'il disait aimer éclate à la fin du roman. La dégradation physique de l'ambassadeur déconnecte tout à fait le sentiment de jalousie d'une situation relationnelle amoureuse, pour laisser la place au seul plaisir pervers d'avilir et de réifier l'autre, de la rabaisser à son rang initial d'esclave, comme pour prouver une dernière fois l'emprise du maître sur l'esclave. Aussi, au terme de sa vie, l'ambassadeur demeure-t-il insensible aux supplications de Théophé de la «délivrer d'une persécution si cruelle», et il la contraint à vivre sous sa tutelle despotique pour le restant de ses jours, non sans manquer de témoigner tout le mépris qu'il éprouve à son égard: 
Là, redoublant tout d'un coup ses pleurs, et se réduisant à des humiliations grecques dont elle devait avoir perdu l'habitude en France, elle se jeta à genoux contre mon lit, pour me supplier de lui accorder ce que je lui avais refusé dans d'autres temps ${ }^{31}$.

Comment prendre conscience du paradoxe de sa conduite? Comment appréhender correctement les causes d'un échec inévitable, puisque la perception qu'il a de l'autre s'oppose précisément à la perception de soi de l'esclave affranchie qui revendique précisément son autonomie? Certain d'être trompé, manipulé, exploité, le narrateur, tout comme le personnage, structure son monde personnel autour de l'idée fixe de la mystérieuse infidèle à laquelle il voue une rancune vengeresse :

[...] je ne pus me livrer si crédulement à l'air de naïveté et d'innocence qu'elle avait su mettre dans sa contenance et dans ses regards. Plus je lui avais reconnu d'esprit, plus je lui soupçonnais d'adresse; et le soin qu'elle avait eu de me faire remarquer plusieurs fois sa simplicité, était précisément ce qui me la rendait suspecte ${ }^{32}$.

Loin d'effectuer un parcours introspectif bénéfique (thérapeutique) qui le libèrerait de ses obsessions, il procède à une mise en récit qui aboutit à une impasse.

La subtile ironie de Prévost à l'égard de ses personnages prétendant à la vérité n'est pas de se moquer, mais de jeter une lumière sur les contradictions psychologiques qui les agitent. Ces contradictions qui obscurcissent la possible ouverture d'une compréhension de soi et de l'autre constituent la substance de l'être humain, sa faiblesse et sa force, sa fragilité et sa puissance. Emblématique à cet égard est la réaction de Cleveland, déjà évoquée, qui évite l’introspection que lui propose son ami, le comte de Clarendon, alors qu'il avait fait serment de ne dire que la vérité:

Il y a peu de personnes qui, dans le récit d'une aventure telle que je vais la rapporter, ne se crussent obligées par amour pour leur réputation d'en déguiser quelques circonstances. Pour moi, qui ai toujours fait profession de croire que le bien ou le mal d'une action doit se tirer du principe qui fait agir, et qu'il n'y a par conséquent que le motif qui déshonore, je n'ai point honte de me laisser voir tel que je suis au public, et de lui faire

31. Prévost, Histoire d'une Grecque moderne, éd. d’Alan. J. Singerman, Paris, Garnier-Flammarion, 1990, p. 290.

32. Ibid., p. 94. 
l'aveu ingénu de mes fautes. C'est assez que je puisse me rendre cet honorable témoignage, que mon cœur a toujours suivi par inclination la vertu et la sagesse; et que s'il s'est trompé quelquefois dans son objet, il n’a jamais manqué de droiture dans ses intentions ${ }^{33}$.

Il est difficile dans ces conditions de savoir si la crise de folie qui pousse le philosophe anglais à l'infanticide et au suicide représente un accès pathologique passager ou un état de folie durable. La crise existentielle située au centre du roman se compose d'une longue réflexion philosophique qui conduit, dans un second temps, le héros éponyme à vouloir mourir avec ses deux fils. Au lieu d'examiner les causes qui l'ont poussé à l'infanticide et au suicide, le héros se tourne du côté des ratiocinations philosophiques. La folie n'est pas représentée dans l'histoire; elle est réfléchie, intégrée dans une pensée philosophique dépressive sur l'existence humaine et le destin calamiteux du personnage qui assume entièrement l'événement, tandis que le je narrateur se contente de rapporter les pensées du personnage et la manière dont celui-ci avait planifié sa folie:

Quoique la résolution que je pris de mourir ne fît que s'affermir à chaque instant, et que je ne trouvasse rien qui s'y opposât dans ma raison, j’eus assez de force pour différer de quelques jours l'exécution de mon dessein. Le motif de ce délai fut tout différent de ce qu'on croirait pouvoir s'imaginer. Je n'avais point d'autre vue que de justifier cette étrange démarche à mes propres yeux par de nouvelles réflexions, et de me convaincre de plus en plus que le ciel même ne la condamnerait point. [...] Ils ne sont pas nés pour être plus heureux que moi. Leur destinée est trop claire. N'eussent-ils à craindre que la contagion de mes infortunes, ils doivent s'attendre à une vie triste et misérable. Quel meilleur office puis-je donc leur rendre que de leur fermer l'entrée d'une carrière de douleurs en terminant leurs jours par une prompte mort. Ils passeront avec moi à une condition plus heureuse. Ils mourront avec leur père. Si je regarde la mort comme un bien, pourquoi ferais-je difficulté de le partager avec mes chers enfants ${ }^{34}$ ?

La scène de folie est racontée par le personnage qui, au moment d'attenter à sa vie, réfléchit aux multiples conséquences d'un acte meurtrier, hésite, s'interroge, se tourmente. C'est d'ailleurs le discours rapporté du personnage qui clôt l'événement: «Étant demeuré seul,

33. Prévost, Le philosophe anglais, op. cit., p. 288.

34. Ibid., p. 289-291. 
je rappelai tout ce qui venait de m'arriver. Je fus d'abord incertain si je devais en remercier le ciel comme d'une faveur, ou me le reprocher comme une faiblesse ${ }^{35}$. $»$ Le narrateur se fond dans la parole du personnage à un moment crucial du récit où le lecteur aurait pu tirer un plein profit des avantages du double registre. Non assumée dans le passé, la folie ne peut être assumée dans le présent de l'écriture, or, elle ne l'est pas. Il est donc impossible de savoir si la guérison a eu lieu ${ }^{36}$. Enfin, après avoir renoncé au meurtre et caché son épée, Cleveland affecte un calme permettant de soulager les soupçons de sa belle-sœur et de Mme Lallin, de sorte que son désordre, écrit-il, «n’a jamais été connu que de [s]es enfants, qui en ont toujours conservé le souvenir, et de Mylord comte de Clarendon ». Un secret bien gardé qu'il enfouit dans les tréfonds de sa mémoire, au moment précis où le Clevelandnarrateur évoque comme on l'a vu le devoir du mémorialiste de ne rien cacher. À la fin de l'épisode, il souligne en caractère gras l'exposition de ses plus «intimes secrets» au public ${ }^{37}$. Pourtant, le secret révélé ne dit rien d'autre qu'une folie spectaculaire, philosophique, pensée et conceptualisée, sans indiquer l'état présent du narrateur qui écrit ses malheurs ni évoquer les leçons tirées d'une telle épreuve.

Pour Jean Sgard, Le philosophe anglais est « une œuvre religieuse ${ }^{38}$ ». Sans doute pourrions-nous ajouter qu'il s'agit d'« une œuvre religieuse manquée », car si Cleveland, à la recherche du bonheur, s’instruit du catholicisme et du protestantisme, il ne réussit pas à trouver un refuge réconfortant dans la foi. L'état de mélancolie dans lequel vit le personnage et qu'il affiche d'entrée de jeu, invite le lecteur à une prise de distance critique par rapport au récit à venir; un récit qui peut être défini comme une cure thérapeutique dans la mesure où le narrateur lui-même se dit malade. Et le long d'une quête tumultueuse, le dilemme entre la nature et la grâce demeure douloureux parce qu'insoluble. L'impact du pyrrhonisme de Pierre Bayle sur la pensée de Prévost apparaît ici, comme le souligne Jean Sgard ${ }^{39}$. Dans cette

35. Ibid., p. 292.

36. Je renvoie à l'article de Florence Magnot, «De la folie aveugle à la folie spectacle: double registre et théâtralité dans les Mémoires et aventures d'un homme de qualité et dans Cleveland de l'Abbé Prévost ", dans René Démoris et Henri Lafon (éd.), Folies romanesques au siècle des Lumières, Paris, Desjonquères, 1998, p. 237-251.

37. Prévost, Le philosophe anglais, op. cit., p. 293.

38. Jean Sgard, Prévost romancier, op. cit., p. 215.

39. Ibid., p. 222. 
perspective, le secret remplit un rôle essentiel. À la fois élément d'ordre (pour qu'une société soit viable, un degré d'opacité est nécessaire) et vecteur de chaos (la nature tourmentée de l'individu comble bien souvent les fissures de sa psyché par un replâtrage fait de secrets et de mensonges), le secret, nous dit Prévost, fait partie de la condition humaine, il doit être pris en compte pour la comprendre.

\section{Conclusion}

L’œuvre de Prévost pose la question de l'identité du moi au centre de la scène romanesque ${ }^{40}$. Mais le désir de connaissance de soi se heurte à des obstacles qui sont pour la plupart reliés à des mensonges, des secrets et des non-dits. Ce bref parcours analyse la place du secret dans un univers romanesque habité par des héros psychologiquement fragiles, qui vivent des conflits insolubles, se débattent avec leurs propres contradictions. Le secret participe également de la construction des relations qui se tissent entre un moi passé et un moi présent, d'une part, et entre un individu et l'autre, qu'il soit l'amant ou l'épouse, la fille ou le frère, d'autre part. À bien des égards, le secret relève d'un désir d'emprise, comme on a pu le voir avec les trois héros narrateurs que j’ai privilégiés dans l'étude présente. L'impasse communicationnelle provoque des failles identitaires qui éloignent le sujet d'une dynamique de réciprocité vitale. Le secret apparaît ici comme l'une des formes principales de la répétition dans son mouvement compulsif. En tant que soustraction à la connaissance humaine en même temps qu'il participe de la connaissance, le secret témoigne, par son usage riche et nuancé, de la capacité du roman prévostien d'absorber plusieurs couches de significations souvent conflictuelles, parfois contradictoires, au sein d'une contre-enquête sur le «moi». Une contre-enquête qui fragilise l'idée que le hasard serait le pivot narratif de ses romans ${ }^{41}$. Une contre-enquête qui met en doute la capacité du sujet de se reconnaître pleinement et se détourne des grands systèmes philosophiques ainsi que des dogmes religieux.

40. Un questionnement qui prend sans doute source dans sa formation théologique (voir A. J. Singerman, op. cit.), mais qui, dès ses premiers écrits, interpelle l'actualité du siècle et participe des débats philosophiques sur l'homme, sa nature et son devenir social.

41. A. B. Maurseth, «Par le biais de la tricherie. Le jeu et le hasard dans Manon Lescaut", dans C. Bournonville, C. Duflo, A. Faulot et S. Pelvilain (éd.), op. cit., p. $129-140$. 